\section{Response to Demerath and Fields}

doi:10.1038/pr.2012.79

To the Editor: We appreciate the feedback provided by Drs Demerath and Fields (1) and the opportunity to respond to concerns they have raised with our publication. First, we studied 6-48 mo olds using the air-displacement plethysmography (ADP) device because infants in our longitudinal studies exceeded US Food and Drug Administration limit of the infant ADP device $(18 \mathrm{~kg})$ in the $4-6$ mo range and we required a way of measuring body composition throughout early childhood for our longitudinal studies. The Food and Drug Administration Medical Device database lists the ADP device as approved for individuals 18 y and older (http://www.accessdata.fda.gov/ cdrh_docs/pdf6/K060848.pdf), so special permission was obtained from our institutional review board to use the device in the age range of our study participants. Research from multiple independent laboratories is needed to determine whether the Pediatric Option for the BOD POD can fill the gap between the infant ADP device and that for adults.

The authors raise concerns about the effect of crying on measurement validity. In fact, to prevent child distress, very few children in our study were tested while crying. However, any loud vocalizations (such as singing or talking loudly to parents) were given a high vocalization score according to our protocol. Considering the high rates of distress (40\%) during testing in the Fields study (2) and the frequency of our observations of loud vocalizations, we believe that a developmentally appropriate method for body fat testing will have to be robust enough to tolerate the variety of behaviors observed in young children in order to be useful in research and clinical settings. This same belief was put forward in the original validation paper for the infant ADP device (3), which specifically ensured that infant crying, urination, and defecation would not affect the accuracy of results, as these developmentally normative behaviors were unavoidable during test conditions.

Demerath and Fields assert that we have not followed typical testing protocol as recommended by the manufacturer. We outline in the methods of our article that a valid "test" included three repeated body volume measurements, consistent with the manufacturer guidelines. If children did not complete all three volume measurements needed for a "test," the data were not used in our analyses. To clarify, $76 \%$ of children completed two repeated "tests" of three body volume measurements and $14 \%$ of children completed only one "test" of three body volume measurements.

Demerath and Fields disagree with our choice of hydration coefficients used to translate total-body water values from our gold standard method, deuterium dilution, into a twocomponent model of body composition (fat mass, fat-free mass) for comparison with ADP. We selected Butte et al.'s (4) hydration coefficients as we believe they are the result of higher-quality studies using more technologically advanced methods than those of Fomon et al. (5). We believe that the Fomon article lacks clear descriptions of the methods used to collect the data and the assumptions involved in constructing the body composition models. In contrast, Butte et al. (4) provide these necessary details. We used the Fomon data for children between 25 and 48 mo of age, as the reference data compiled by Butte et al. (4) describe only children up to 2 y of age.

Finally, Demerath and Fields claim the four component (4-C) model is "superior to any single method" as a gold standard. We strongly disagree with that assertion as applied to both our research article and Fields' recently published evaluation of ADP in children age 2-6 y (2). The 4-C model is an excellent gold standard when evaluating a test method that is "independent" of the measurements contributing to the 4-C model (density, water, and mineral). To illustrate why, consider data from a study of wrestlers published by Clark et al. (6) comparing body composition measurements from hydrostatic weighing (the source of the density value in the 4-C model) to the 4-C criterion. The two methods were correlated at a rate of 0.98 ; thus, more than $96 \%$ of the variance in the 4-C model was attributed to the density value originating with hydrostatic weighing. In Fields' study (2), any errors in the ADP measurement of body density would result in aberrant values for their criterion method, and more significantly, these errors could not be detected! With such a research design, it is nearly impossible to find that ADP is inaccurate, which we believe is unacceptable for evaluating the accuracy, precision, and trueness of any new test method. Although deuterium dilution is not a perfect gold standard, nevertheless it is clearly preferable to an ADP-based 4-C model in young children, and we maintain that having a truly independent measure of body composition is the only acceptable way in which to generate a comparison with ADP values.

Although we appreciate the concerns raised by Demerath and Fields, we disagree that the issues they raise are responsible for the poor agreement of ADP with our gold standard method. The precision data we present on phantoms confirm our assertion that ADP's inaccuracy is more likely due to difficulties in measuring small volumes, rather than child behavior or the choice of hydration coefficients and gold standard techniques. This finding also is supported by the research of Collins and McCarthy (7), who noted a decrease in precision in ADP volume measurements of phantoms less than $40 \mathrm{l}$. Collins and McCarthy (7) postulated that the observed discrepancies may be caused by a high chamber to body volume ratio, a theory supported by our precision data.

In sum, we share the overarching goal expressed by Demerath and Fields - that the field of child obesity research 


\section{Correspondence}

will identify one or more methods that accurately, quickly, and painlessly measure body composition of young children. The results of our study, which received no industry funding, suggest that ADP is not yet meeting that goal in children aged 6-48 mo.

\section{Ryan P. Rosendale ${ }^{1}$ and Cynthia J. Bartok ${ }^{2}$}

${ }^{1}$ Department of Kinesiology, The Pennsylvania State University, University Park, Pennsylvania; ${ }^{2}$ Resource Center for the Prevention of Military Child Obesity, Clearinghouse for Military Family Readiness, The Pennsylvania State University, University Park, Pennsylvania. Correspondence: Ryan P. Rosendale (rpr135@psu.edu)

\section{REFERENCES}

1. Demerath EW, Fields DA. Challenges in infant body composition. Pediatr Res 2012; 72:329.
2. Fields DA, Allison DB. Air-displacement plethysmography pediatric option in 2-6 years old using the 4-compartment models as a criterion method. Obesity (Silver Spring), 2012; e-pub ahead of print 15 March 2012.

3. Ma G, Yao M, Liu Y, et al. Validation of a new pediatric air-displacement plethysmograph for assessing body composition in infants. Am J Clin Nutr 2004;79:653-60.

4. Butte NF, Hopkinson JM, Wong WW, Smith EO, Ellis KJ. Body composition during the first 2 years of life: an updated reference. Pediatr Res 2000;47:578-85.

5. Fomon SJ, Haschke F, Ziegler EE, Nelson SE. Body composition of reference children from birth to age 10 years. Am J Clin Nutr 1982;35 (5 Suppl):1169-75.

6. Clark RR, Bartok C, Sullivan JC, Schoeller DA. Minimum weight prediction methods cross-validated by the four-component model. Med Sci Sports Exerc 2004;36:639-47.

7. Collins AL, McCarthy HD. Evaluation of factors determining the precision of body composition measurements by air displacement plethysmography. Eur J Clin Nutr 2003;57:770-6. 